\title{
Alternativa de Convivencia con el Problema de la Salinidad del Agua de Irrigación causada por Presas Subterráneas
}

\author{
José Wilmar da S. Neto, Carisia C. Gomes, Ernesto da S. Pitombeira y Ramyro B. Araújo \\ EMLURB-Empresa Municipal de Limpeza e Urbanização, Prefeitura Municipal de Fortaleza, \\ German Alumni Water Network, Fortaleza - CE, 60030-141, Brasil \\ (e-mail: jwilmarsn@gmail.com)
}

Recibido Jun. 09, 2010; Aceptado Ago. 12, 2010; Versión Final recibida Ago. 24, 2010

\begin{abstract}
Resumen
Se presenta una alternativa de convivencia con el problema de salinidad del agua de irrigación causado por presas subterráneas. Se analiza la calidad del agua de irrigación comparando el crecimiento vegetativo de las gramíneas Capim Vetiver; Sempre Verde; Cana-de-açúcar; Capim Elefante cv. Napier y Capim Elefante cv. Mercker, aguas arriba y abajo de la presa subterránea localizada en el municipio de Ibicuitinga, estado de Ceará en Brasil. El análisis de muestras de aguas subterráneas aguas arriba y abajo de la presa han mostrado que ambas poseen un grado de salinidad elevado para fines de irrigación, recomendándose el uso de cultivos halófitos. Se ha realizado el análisis de varianza para variable crecimiento en altura y se ha aplicado la prueba de Tukey. A partir de esta prueba se ha verificado que, de entre las gramíneas probadas, tan sólo la Capim Elefante cv. Mercker ha diferido estadísticamente.
\end{abstract}

Palabras clave: aguas subterráneas, salinidad, especies halófitas, crecimiento de gramíneas

\section{Alternative of Coexistence with the Salinity Problem of Irrigation Water caused by Underground Dams}

\begin{abstract}
The objective of this paper is to show an alternative for the coexistence with the salinity problem of irrigation water caused by underground dams. The quality of irrigation water is analyzed by comparing the vegetative growth of Capim Vetiver, Sempre Verde, Cana-de-Açúcar, Capim Elefante cv. Napier and Capim Elefante cv. Mercker, upstream and downstream of the underground dam located in Ibicuitinga, Ceará state in Brazil. The analysis of groundwater samples upstream and downstream of the dam have shown that both have high salinity for irrigation, recommending the use of halophytes crops. The study includes an analysis of variance for height growth variable and the Tukey test was applied. From this test it was verified that among the gramineous plants tested, only the Capim Elefante cv. Mercker was found statistically different.
\end{abstract}




\section{INTRODUCCIÓN}

El Nordeste semiárido de Brasil posee un elevado número de presas superficiales, desde las de gran porte como Orós y Castanhão, en el estado de Ceará, y Armando Ribeiro Gonçalves, en el estado de Rio Grande do Norte, con acumulación de billones de metros cúbicos de agua, hasta las pequeñas presas, que acumulan miles de metros cúbicos y, en general, no soportan, sin secar, un período anual de sequía, gracias a la elevada tasa de evaporación nominal media de,

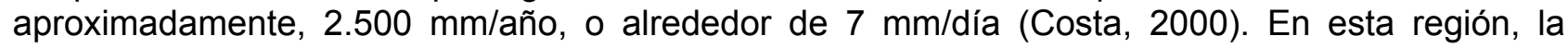
inestabilidad climática se ve más afectada por su irregularidad que por la escasez, constituyendo un gran obstáculo para la permanencia del hombre en el medio rural, debido a la falta de agua incluso para suplir sus necesidades básicas. Como en otras regiones semiáridas del mundo, el trópico semiárido brasileño presenta también suelos rasos y pedregosos, con baja capacidad de retención de agua, bajo contenido de materia orgánica y alta potencialidad para la erosión (Cirilo y Costa 2006). Según Anjos et al. (2007), el problema de la región Nordeste es el de la heterogeneidad en la distribución de los acuíferos. Solamente dos estados disponen de agua subterránea, supliendo sus demandas por ellos mismos. Son los estados de Piauí y Maranhão. "En los demás, el agua se concentra en determinadas zonas y, sobre todo, en el interior, en el semiárido cristalino (un 55\% del área de la región), el agua está en pequeña cantidad y en caudales muy bajos, en general, de elevada salinidad, ya que la evaporación es muy elevada con relación a la precipitación". Existen diferentes alternativas para la creación y explotación de reservas hídricas en esa región. Los depósitos superficiales son los más utilizados, debido a las condiciones geológicas que favorecen un elevado desagüe superficial. La creación de acuíferos artificiales (a través de la construcción de presas subterráneas) permiten almacenar agua con calidad y en cantidad para suplir las necesidades de una familia o comunidad, de los animales e incluso de una pequeña irrigación (Rebouças, 1999). La construcción de presas subterráneas es una tecnología alternativa de captación de agua de lluvia, de construcción fácil y de bajo coste, permitiendo cultivar cultivos tradicionales, producir fructíferas y asegurar el abastecimiento de agua para animales (en determinadas circunstancias, incluso para consumo humano) a través de la excavación de pozos en plena área de vegetación llamada caatinga, a partir de la humedad proporcionada por el agua de lluvia captada y contenida en un área determinada, contribuyendo así a la mejora de la calidad de vida de las familias en el semiárido (Silva et al., 2008). A pesar de la simplicidad, la construcción de la presa subterránea exige algunos requisitos técnicos. Debe elegirse adecuadamente su ubicación, lo que no significa decir que su instalación tenga que darse en el lecho del río o riacho, sino que es imprescindible que se implante en un punto estratégico del terreno donde desagüe el mayor volumen de agua en el momento de lluvia (Chaves et al., 2007). El uso de estas aguas bajo condiciones adecuadas de manejo no debe ofrecer mayor problema para la irrigación. Sin embargo, esa región presenta una elevada predisposición a la degradación oriunda del uso incorrecto de prácticas agrícolas posiblemente adoptadas que, unida a la lixiviación incompleta y a la intensa evaporación, resultan en un aumento de las sales en las capas superficiales del suelo debido al balance inadecuado de sales comúnmente verificado, aumentando progresivamente las áreas problemáticas (Ferreira et al., 2008). Por otro lado, la práctica de la irrigación en áreas de dominio de acuíferos aluviales rasos, asociados a las condiciones climáticas y edáficas desfavorables, puede incurrir en impactos ambientales adversos al suelo y al agua subterránea, ocasionando la concentración excesiva de sales (Mendonça et al., 2007). De acuerdo con Miranda (2000), el uso de métodos biológicos para recuperación de suelos salinos, aunque sea bastante prometedor, actualmente se presenta bastante limitado en virtud de la pequeña disponibilidad de especies de interés comercial, por el bajo nivel de tolerancia de las plantas al estrés salino y por la poca disponibilidad de datos que puedan orientar su cultivo. De esa forma, debido a la cuestionable eficacia de los métodos tradicionales de recuperación de suelos salinos, la adaptación de las plantas a esos ambientes se configura como una salida prometedora.

Con base en lo expuesto, el presente trabajo tiene como objetivo mostrar cómo se puede convivir con el problema de salinidad del agua represada en presas subterráneas analizando la calidad del agua de irrigación y su relación con el desarrollo, o el crecimiento vegetativo, de gramíneas introducidas en las áreas saturadas aguas arriba y abajo de la presa subterránea, localizada en el río Palhano, municipio de Ibicuitinga, estado de Ceará, Brasil. Con base en la observación y 
comparación de la producción de las especies vegetales y en los resultados del análisis del agua, se ha optado por trabajar con las siguientes gramíneas: Capim Vetiver (Vetiveria zizanioides); Sempre-Verde (Panicum maximum); Cana-de-açúcar (Saccharum officinarum L.); Capim Elefante cv. Napier (Pennisetum purpureum Schum) y Capim Elefante cv. Mercker (Pennisetum purpureum Schum).

\section{MATERIAL Y MÉTODOS}

\section{Descripción del área de estudio}

La investigación se ha desarrollado en la presa subterránea del río Palhano, municipio de Ibicuitinga, estado de Ceará, región Nordeste de Brasil (Figura 1). Esa región pertenece al semiárido, poseyendo características preponderantes tales como vegetación caatinga y escasez de recursos hídricos debido a la irregularidad de las precipitaciones pluviométricas y poco almacenamiento de agua.

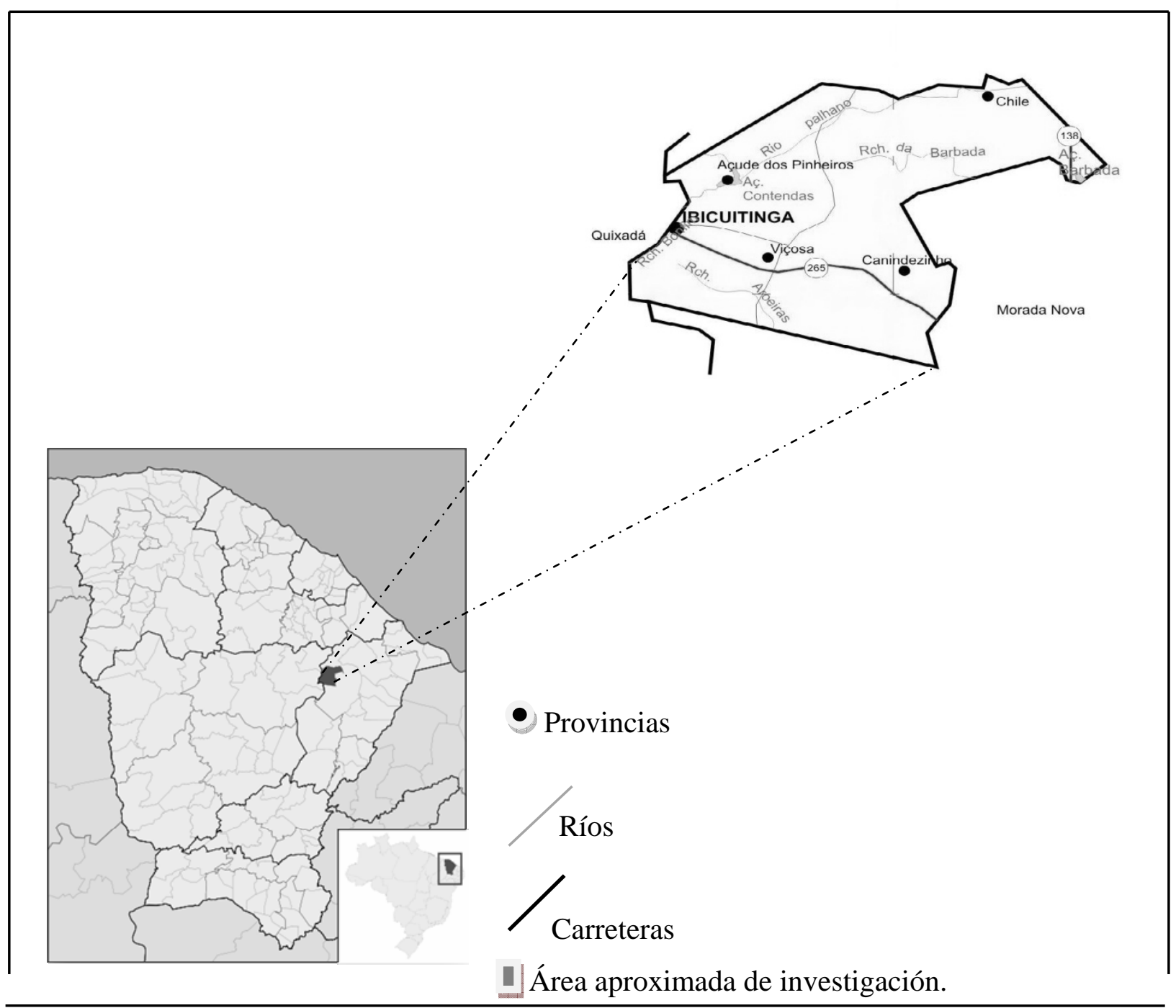

Fig. 1: Mapa de localización del área de investigación en el municipio de Ibicuitinga, Ceará, Brasil.

Según Gomes (1990), la presa del río Palhano, afluente de la orilla izquierda del río Jaguaribe, está limitada aguas arriba por la presa Chile, lo que garantiza la recarga de agua periódica, proporcionando una renovación de cantidad y calidad de los volúmenes almacenados. La cuenca hidrográfica que contiene el área de investigación se localiza entre las coordenadas $4^{\circ} 50^{\prime}-4^{\circ} 58^{\prime}$ sur de latitud y $38^{\circ} 26^{\prime}-38^{\circ} 39^{\prime}$ este de longitud y posee un área igual a $175,00 \mathrm{Km}^{2}$. Según Monteiro (1988), esta presa subterránea se constituye en un diafragma impermeable de suelo compactado transversal al río, asentado sobre estrato rocoso, elevado hasta que la cresta de la 
presa alcanza la cota del lecho del río, con la finalidad de interceptar el flujo de agua subterránea proporcionando la elevación del nivel freático y, consecuentemente, el almacenamiento de volúmenes de agua en los vacíos intersticiales de los suelos, sin interrumpir el desagüe superficial del río, atendiendo así a la necesidades básicas de abastecimiento de agua para consumo humano y animal.

\section{Instalación y conducción del experimento}

Los trabajos de investigación en la presa se han iniciado con la limpieza del área, cercamiento y excavación manual de dos pozos artesianos, con la recogida de suelo y, a continuación, la recogida periódica de muestras de agua para realización del análisis de laboratorio de las mismas, tomando como base los estándares de Embrapa (1997) y Apha (1992), respectivamente. Los pozos artesianos se han construido utilizándose anillos de hormigón con un diámetro de 1,20 $\mathrm{m}$ y profundidad variable de 2,00 a 2,50 m, situándose uno aguas arriba y otro aguas abajo de la presa subterránea. La finalidad de los mismos es la de proporcionar puntos de recogida de agua para irrigación aguas arriba y abajo respectivamente. La elección de las especies de la mayoría de las gramíneas se ha realizado, principalmente, debido al hecho de que las mismas han nacido y crecido espontáneamente en áreas salinizadas cercanas a la presa subterránea de Ibicuitinga y también con base en las siguientes características: ser plantas pertenecientes a la vía fotosintética C4 de la familia de las gramíneas y poseer follaje apreciado por animales domésticos y de la fauna. Excepto el Capim Vetiver, que se ha introducido por haber sido citado en mucha literatura como planta de carácter halófito, es decir, resistente a las condiciones extremas de salinidad. A continuación se han empezado a plantar las siguientes gramíneas: Capim Vetiver (Vetiveria zizanioides); Sempre-Verde (Panicum maximum); Cana-de-açúcar (Saccharum officinarum L.); Capim Elefante cv. Napier (Pennisetum purpureum Schum) y Capim Elefante cv. Mercker (Pennisetum purpureum Schum). Se han plantado 15 unidades de cada especie, con un espacio de $50 \mathrm{~cm}$ en canteros de $5 \mathrm{~m}^{2}$, tanto aguas arribas y abajo de la presa subterránea, para garantizar que por lo menos 10 de ellas sobreviven para realizar el acompañamiento semanal de crecimiento de las mismas. Tras plantar las gramíneas, se ha ampliado el aprovechamiento de las áreas limítrofes de la presa, implantando como cerca viva el Pinhão bravo (Jatropha molissima), por ser una planta nativa y abundante en la caatinga de la región, rústica, perenne, adaptable a una amplia gama de ambientes y condiciones edafo-climáticas, tolerante a la sequía, poco atacada por plagas y enfermedades y principalmente por tener potencial de producción de biodiesel. La cerca viva tiene las funciones de cerrar y aislar el área de la presa subterránea, protegiéndola de la invasión de animales, y mejorar la calidad del agua que llega a la presa, tanto por la contención de sedimentos como por el filtrado del agua a través de las raíces y la contención de la erosión.

Siguiendo las recomendaciones de Almeida (2009), la producción de las mudas de Pinhão bravo para el experimento se ha hecho a través de estacas, las cuales se han extraído de ramas leñosas, plantas con buena formación, buenas producciones, perfecto estado sanitario y libre de plagas y enfermedades. La longitud de las mudas ha sido de 1 metro, con diámetro medio de 5 $\mathrm{cm}$. Siguiendo sugerencias de Saturnino et al. (2007), las mudas se han enterrado en el suelo por la base a una profundidad de $20 \mathrm{~cm}$, con un espacio de $1 \mathrm{~m}$ en los bordes de la presa para proponer su uso en cerca viva. Se ha intentado trabajar con estacas uniformes teniendo en cuenta las características morfológicas externas. Con base en las sugerencias de Silveira Neto (2003), se han seleccionado los parámetros de crecimiento en altura y la observación visual de síntomas de quema de hojas, para acompañamiento semanal de las características de desarrollo vegetativo de las gramíneas del estudio. La monitorización del desarrollo vegetativo de esas plantas se ha hecho semanalmente durante un período de 8 meses. Durante el experimento se han medido los niveles de sales presentes en las aguas arriba y abajo de la presa a través de análisis físicoquímicos de los mismos. Esos análisis han permitido calcular los contenidos de salinidad de las aguas con base en los parámetros de Conductividad Eléctrica (CE) y Razón de Absorción de Sodio (RAS), sugeridos por el laboratorio de salinidad de Estados Unidos (Bernardo, 2006). Se han seleccionado aleatoriamente 3 plantas de cada fila de cada especie para medición de sus alturas durante el tiempo de investigación y finalmente se han calculado las medias para cada especie estudiada. Este procedimiento se ha repetido 17 veces a lo largo del período de recogida 
de datos. Ya con todos los datos, se ha realizado el análisis estadístico de varianza para variable crecimiento en altura. Posteriormente, cuando significativo por la prueba $\mathrm{F}$ al nivel de $5 \%$ de probabilidad, se ha aplicado la prueba de Tukey al nivel de $5 \%$ de probabilidad. Para la realización de estos análisis, se ha utilizado el software "SAEG 9.0 - UFV".

\section{RESULTADOS Y DISCUSIÓN}

Para el análisis de la calidad de las aguas arriba y abajo de la presa subterránea ha sido necesario monitorizar la misma a lo largo del período de estudio. La Tabla 1 presenta el resultado del análisis físico-químico del agua para ambos lados de la presa del río Palhano y la clasificación de las mismas según los parámetros de salinidad. Según la Tabla 1, se han escogido los parámetros Conductividad Eléctrica (CE), Razón de Absorción de Sodio (RAS) y Potencial de Hidrógeno $(\mathrm{pH})$. La Conductividad Eléctrica (CE) es un buen indicador de la cantidad total de sales en el agua. Cuanto mayor sea su valor, mayor será la cantidad de sales que cuando se acumulen en el suelo reducirán el rendimiento de los cultivos (Ayers \& Westcott, 1991). Según la Tabla 01, la media de la conductividad eléctrica ha presentado valores de $1,70 \mathrm{dSm}^{-1}$ y $3,58 \mathrm{dSm}^{-1}$ respectivamente aguas arriba y abajo de la presa. Según Reichart (1978), con relación a la CE, las aguas de calidad comprendidas entre 0,75 a 2,25 $\mathrm{dSm}^{-1}$ presentan un alto riesgo de salinidad, debiendo recibir tratamiento especial para que pueda utilizarse y las aguas que presentan una conductividad entre 2,25 y $5,0 \mathrm{dSm}^{-1}$ presentan un riesgo muy alto de salinidad. De esta forma, esas aguas no son adecuadas para irrigación común. Necesitan que los suelos sean permeables, bien drenados, para que haya un exceso de lixiviación y puedan utilizarse con cultivos halófitos.

Tabla 1: Clasificación físico-químico de las aguas arriba y abajo para fines de irrigación, recogida a lo largo de la investigación en Ibicuitinga, Ceará.

\begin{tabular}{ccccccccc}
\hline & \multicolumn{7}{c}{ MUESTRAS } \\
\cline { 2 - 8 } PARÁMETROS & $20 / 09 / 2007$ & $23 / 01 / 2008$ & $28 / 02 / 2008$ & $25 / 04 / 2008$ \\
\hline Cationes $\left(\mathrm{mmol}_{\mathrm{c}} \mathrm{L}^{-1}\right)$ & $\mathrm{Ar}$ & $\mathrm{Ab}$ & $\mathrm{Ar}$ & $\mathrm{Ab}$ & $\mathrm{Ar}$ & $\mathrm{Ab}$ & $\mathrm{Ar}$ & $\mathrm{Ab}$ \\
$\mathrm{Ca}^{++}$ & 5,0 & 6,0 & 3,1 & 3,9 & 2,2 & 3,8 & 1,3 & 2,6 \\
$\mathrm{Mg}^{++}$ & 4,5 & 5,0 & 2,9 & 3,1 & 2,5 & 3,3 & 0,6 & 1,9 \\
$\mathrm{Na}^{+}$ & 14,3 & 38,0 & 13,6 & 34,8 & 14,6 & 32,1 & 2,3 & 8,3 \\
$\mathrm{~K}^{+}$ & 0,3 & 0,3 & 0,4 & 0,2 & 0,3 & 0,1 & 0,2 & 0,2 \\
$\Sigma$ & 24,1 & 49,3 & 20,0 & 42,0 & 19,6 & 39,3 & 4,4 & 13,0 \\
$\sum_{\text {Aniones }\left(\mathrm{mmol}_{\mathrm{c}}{ }^{-1}\right)}$ & 20,4 & 44,0 & 16,0 & 36,0 & 16,4 & 32,0 & 3,4 & 11,0 \\
$\mathrm{Cl}^{-}$ & 4,0 & 4,8 & 3,8 & 5,6 & 3,2 & 7,6 & 1,0 & 2,0 \\
$\mathrm{HCO}_{3}^{-}$ & 24,4 & 48,8 & 19,8 & 41,6 & 19,6 & 39,6 & 4,4 & 13,0 \\
$\Sigma$ & 2,43 & 4,86 & 1,98 & 4,17 & 1,98 & 3,97 & 0,4 & 1,30 \\
$\mathrm{CE}\left(\mathrm{dSm}^{-1}\right)$ & 6,58 & 16,2 & 7,83 & 18,59 & 9,38 & 17,15 & 2,44 & 5,61 \\
$\mathrm{RAS}_{\mathrm{pH}}$ & 6,7 & 7,4 & 6,8 & 7,2 & 6,7 & 7,1 & 7,3 & 7,4 \\
Shólidos disueltos $\left(\mathrm{mgL}^{-1}\right)$ & 2430 & 4860 & 1980 & 4170 & 1980 & 3970 & 440 & 1300 \\
\hline Clasificación & $\mathrm{C} 4 \mathrm{~S} 2$ & $\mathrm{C} 4 \mathrm{~S} 4$ & $\mathrm{C} 3 \mathrm{~S} 2$ & $\mathrm{C} 4 \mathrm{~S} 4$ & $\mathrm{C} 3 \mathrm{~S} 2$ & $\mathrm{C} 4 \mathrm{~S} 4$ & $\mathrm{C} 2 \mathrm{~S} 1$ & $\mathrm{C} 3 \mathrm{~S} 1$ \\
\hline
\end{tabular}

Ar - Arriba; Ab - Abajo

La Figura 2 muestra un gráfico con el análisis de la CE a lo largo del período del experimento en la presa subterránea en Ibicuitinga, Ceará. En este gráfico, se nota la disminución de la salinidad del agua en la presa subterránea desde enero de 2008. Esto se debe al hecho de haber empezado el período de lluvias y, como era de esperar, con el aumento de las precipitaciones pluviométricas hay una dilución mayor de esas sales presentes en el suelo y en el agua. Para el cálculo de la RAS se han utilizado los parámetros $\mathrm{Na}^{+}, \mathrm{Ca}^{++}$y $\mathrm{Mg}^{++}$. La dispersión del suelo puede ocurrir cuando los contenidos de calcio son insuficientes para contrarrestar el efecto de dispersión de altos contenidos de sodio que tienden a acumularse en los estratos superficiales. Además, los efectos del exceso de magnesio cambiable no sólo pueden inducir a deficiencia de calcio en las plantas (Bastos, 1999, Bouwer \& Idelovitch, 1988, Metcalf \& Eddy, 1991). La Figura 3 presenta un gráfico con la variación de la RAS en el período de estudio. Se nota también la disminución de la 
RAS del agua en el período de lluvias, también debido al aumento de las precipitaciones pluviométricas y, consecuentemente, la dilución del $\mathrm{Na}^{+}, \mathrm{Ca}^{++}$y $\mathrm{Mg}^{++}$presentes en el suelo y en el agua. Con relación a la calidad del agua, cuando la misma presenta alto contenido de sodio, se desarrolla en el suelo una condición de suelo sódico que disminuye la permeabilidad del suelo al agua. La calidad del agua, con respecto al sodio, se evalúa a través de la RAS, la cual tiene en cuenta el exceso de sodio con relación al calcio y al magnesio. La media de la RAS ha presentado valores de 6,56 y 14,39, respectivamente aguas arriba y abajo de la presa.

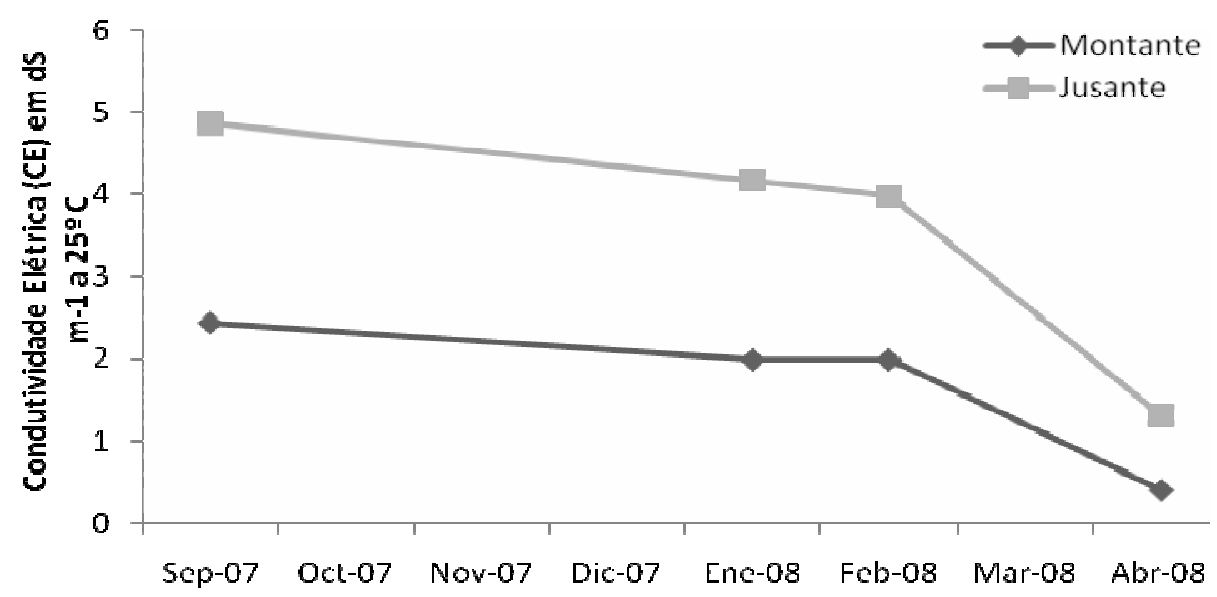

Fig. 2: Análisis de la Conductividad Eléctrica (CE) a lo largo de la investigación en la presa subterránea en Ibicuitinga, Ceará.

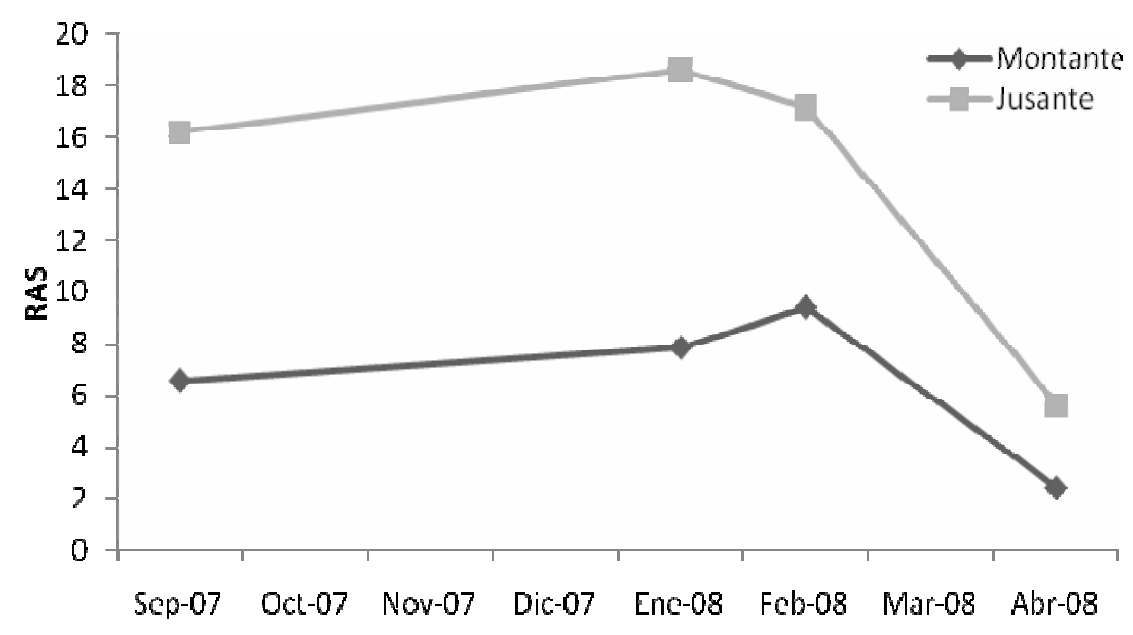

Fig. 3: Análisis de la Razón de Absorción de Sodio (RAS) a lo largo de la investigación en la presa subterránea en Ibicuitinga, Ceará.

Según Reichart (1978), con relación a la RAS, las aguas de calidad comprendidas entre 5,0 y 8,0 presentan un alto riesgo de disminución de permeabilidad y para RAS por encima de 8,0 presentan un riesgo muy alto de disminución de permeabilidad, debiendo evitarse su uso. Con relación al $\mathrm{pH}$, cuando éste esté fuera de los estándares establecidos en el agua de irrigación, puede causar serios problemas a las cultivos, como crecimiento excesivo, maduración tardía, caída del vegetal, producción reducida y de mala calidad e incluso desequilibrio nutricional. Además de estos problemas, también están los relacionados con las enfermedades transmisibles a través del agua de irrigación (Bernardo, 2006). Cuando los valores de pH superan la amplitud normal de 6,5 a 8,4 tiene lugar la disponibilidad de iones tóxicos para las plantas, como cloratos, sodio y boro, donde los daños a los cultivos y al suelo pueden provocarse individualmente o con la combinación de estos iones (Ayers \& Westcott, 1991). La media de pH del agua encontrada en la presa subterránea ha sido de 6,9 y 7,3, aguas arriba y abajo respectivamente, estando dentro de la amplitud recomendada y siendo su uso indicado para irrigación según ese parámetro. Para el estudio del crecimiento de las gramíneas se ha utilizado el gráfico Boxplot (Figura 4), que tiene el 
conjunto de medidas estadísticas, ofreciendo la idea de la posición, dispersión, asimetría, caudales y datos discrepantes.

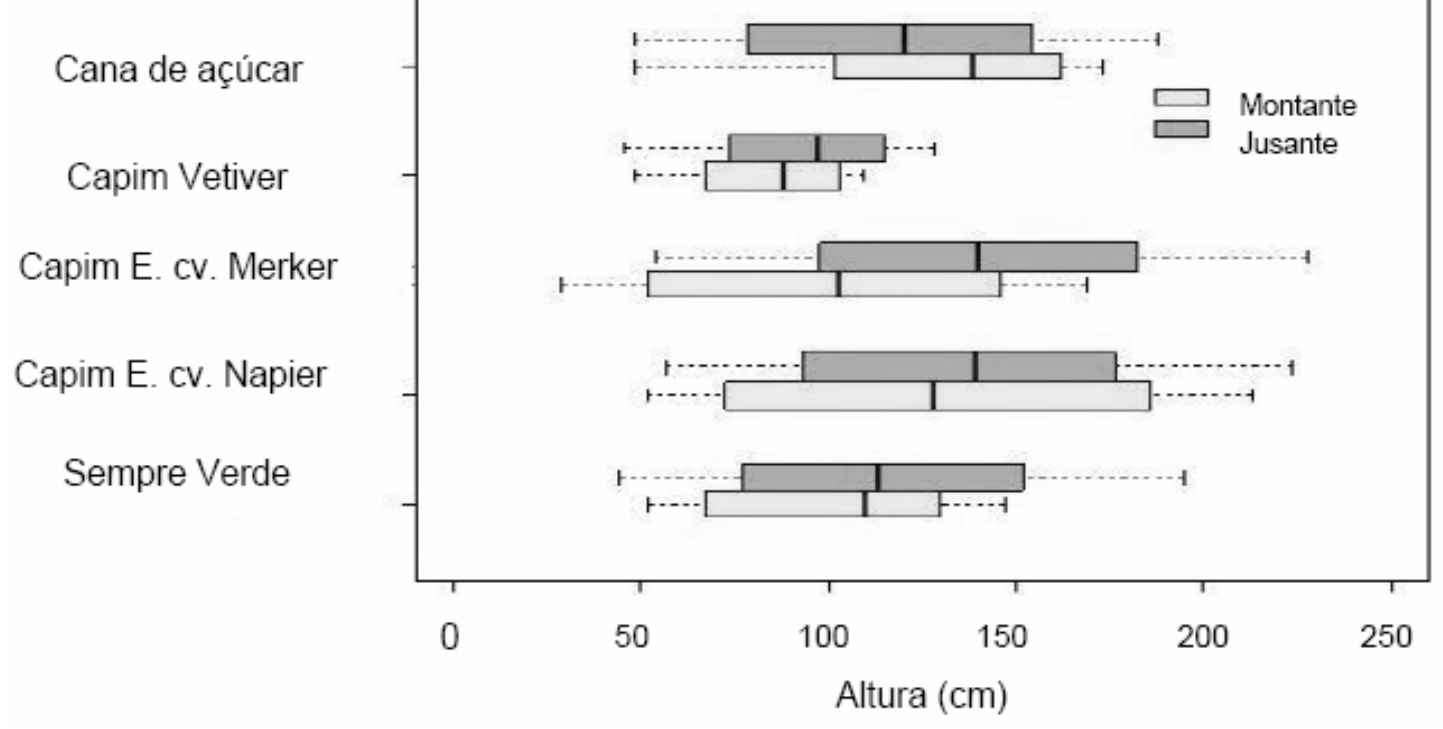

Fig. 4: Gráfico Boxplot entre los tratamientos de las gramíneas aguas arriba y abajo de la presa subterránea en Ibicuitinga - Ceará.

En la Figura 4 se observa que ha habido interacción entre las variables Tipo de gramínea y Lado de la presa (aguas arriba o abajo). Se nota también que existe heterogeneidad en los datos. Así, ajustándose los datos transformados, se obtiene la Tabla 2 con el resumen del análisis de varianza del experimento.

Tabla 2: Resumen del análisis de varianza para las variables: Gramíneas y tipo de suelo (aguas arriba y abajo de la presa)

\begin{tabular}{cccccc}
\hline Factor de variación & $\begin{array}{c}\text { Grado de } \\
\text { libertad }\end{array}$ & $\begin{array}{c}\text { Suma de } \\
\text { cuadrados }\end{array}$ & $\begin{array}{c}\text { Cuadrado } \\
\text { medio }\end{array}$ & Valor-F & Valor-P \\
\hline Lado de la presa & 1 & 37.651 & 37.651 & 0.613 & 0.439 \\
Error 1 & 32 & 1964.942 & 61.404 & & \\
Gramíneas & 4 & 232.765 & 58.191 & 64.006 & $<0.0001^{* * *}$ \\
Lado de la & 4 & 85.228 & 21.307 & 23.436 & $<0.0001^{* * *}$ \\
presa*Gramíneas & 128 & 116.372 & 0.909 & & \\
Error 2 & & & & \\
\hline
\end{tabular}

*** Significativo al nivel de $5 \%$.

Obs.: Las celdas en blanco no necesitan pruebas estadísticas, ya que se utilizan para aplicación de la estadística de la prueba $\mathrm{F}$.

En la Tabla 2 puede verse que existe interacción significativa, al nivel de significancia de $5 \%$, entre el Lado de la presa y el Tipo de gramínea. Así, se ha utilizado la prueba de Tukey para comparación múltiple de las medias. Esta prueba verifica la igualdad del tratamiento principal cuando se fija el tratamiento secundario, y vice-versa. Se observa que existe efecto del tipo de gramínea, pero por el hecho de existir efecto de interacción, el análisis debe hacerse encima de la propia interacción, es decir, no se puede analizar el efecto aislado del Tipo de gramínea. De acuerdo con la Tabla 3, se ve que cuando se fija las gramíneas en el estudio, la mayoría no tienen respuestas diferentes, es decir, cuando se trabaja con la variable Arriba y la variable Abajo, las gramíneas no difieren estadísticamente entre sí, excepto la gramínea Capim E. cv. Mercker que ha diferido estadísticamente entre sí. 
Tabla 3: Valores medios, por tratamiento, obtenidos para las variables Tipos de gramíneas en función del Lado de la presa (Arriba y Abajo).

\begin{tabular}{cccccc}
\hline \multirow{2}{*}{$\begin{array}{c}\text { Lado de la } \\
\text { presa }\end{array}$} & Sempre Verde & Capim Napier & Capim Mercker & Capim Vetiver & $\begin{array}{c}\text { Cana de } \\
\text { açúcar }\end{array}$ \\
\cline { 2 - 6 } & $101.8235 \mathrm{a}$ & $131.4706 \mathrm{a}$ & $99.5588 \mathrm{a}$ & $83.94118 \mathrm{a}$ & $129.1765 \mathrm{a}$ \\
Arriba & $115.3235 \mathrm{a}$ & $137.5000 \mathrm{a}$ & $140.9705 \mathrm{~b}$ & $92.70588 \mathrm{a}$ & $117.6765 \mathrm{a}$ \\
\hline
\end{tabular}

Letras iguales $(a, a)$ muestran que no ha habido diferencia estadística significativa entre los tratamientos. Letras diferentes $(a, b)$ muestran que sí ha habido diferencia estadística significativa entre los tratamientos usados en el experimento. De esta forma, según la prueba de Tukey, la única especie que difiere estadísticamente es la Capim Elefante cv. Mercker. La Figura 5 ilustra mejor esa comparación entre los lados Arriba y Abajo de la presa subterránea cuando se fija la especie de gramínea.

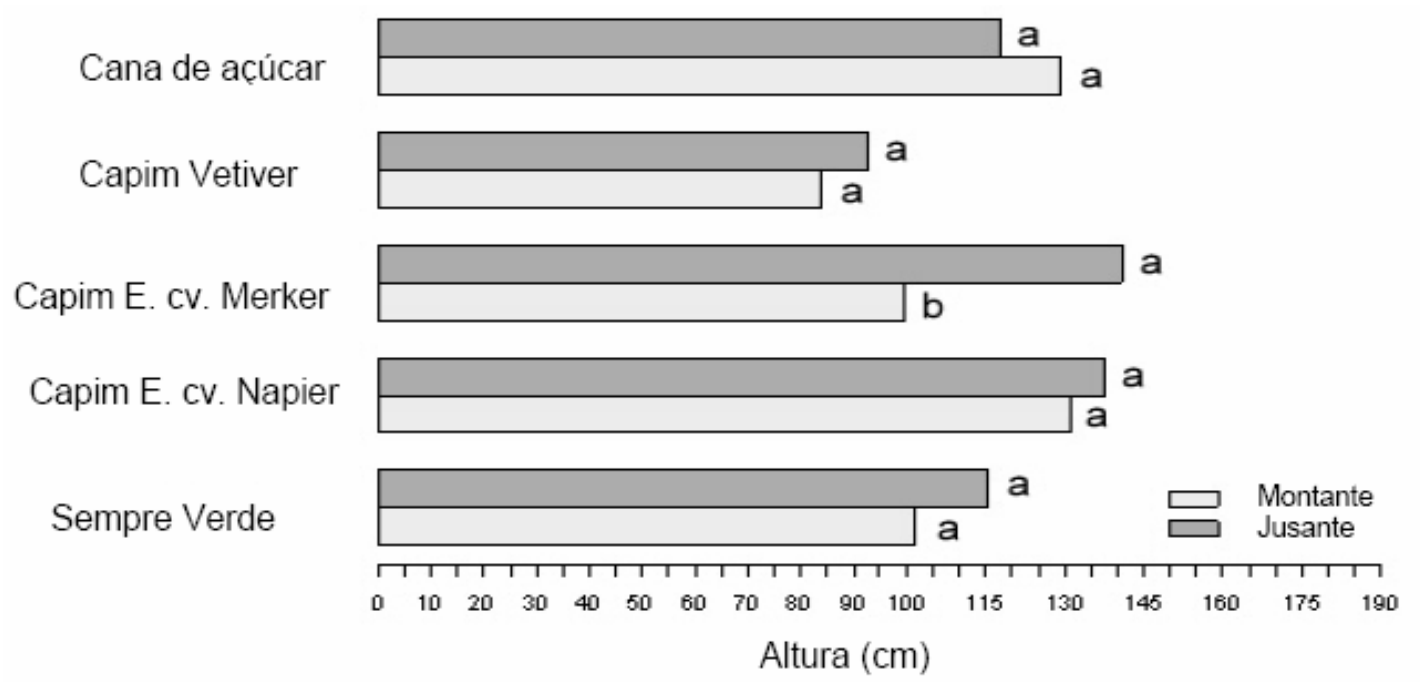

Fig. 5: Representación de la prueba de medias para la variable crecimiento en altura cuando se fija la especie de gramíneas aguas arriba y abajo de la presa subterránea en Ibicuitinga - CE.

A partir de la Figura 5, se entiende que la mayoría de las especies ha crecido mejor aguas abajo de la presa, área que presentaba mayor salinidad. Ese factor refuerza el carácter halófito de las especies probadas. Excepcionalmente, la Cana de açúcar se ha comportado de forma diferente de las demás, desarrollándose mejor aguas arriba, región que posee un ambiente de menor salinidad.

\section{CONCLUSIONES}

La recogida de muestras y el análisis de aguas subterráneas aguas arriba y abajo de la presa han mostrado que ambas poseen salinidad elevada para fines de irrigación. Las medias de los resultados de los análisis han comprobado que el agua de irrigación aguas arriba de la presa presenta un alto riesgo de salinidad y un contenido medio de sodios, siendo clasificadas como C3S2. Para las aguas abajo de la presa, las medias de los resultados de los análisis del agua han mostrado que el agua de irrigación presenta un riesgo muy alto de salinidad y un contenido muy alto de sodios, siendo clasificada como C4S4. La variación de la salinidad del agua de la presa subterránea, con su disminución en el período de lluvias (tasas pluviométricas mayores) se debe, naturalmente, al aumento de la infiltración de agua en el suelo y, consecuentemente, la dilución de las sales presentes tanto en el suelo como en el agua del acuífero. En observaciones en el campo no se ha notado ningún síntoma de intolerancia al sodio de las gramíneas probadas en el área de 
la presa subterránea. Estos síntomas son, por ejemplo, quemaduras en las hojas, atrofia del crecimiento de hojas y tallos, así como otros factores que indicasen esa posibilidad, hecho que constata el carácter halófito de las plantas probadas. La investigación ofrece una alternativa posible de convivencia con las condiciones salinas del agua y del suelo, causada por la implantación de presas subterráneas con la utilización de gramíneas halófitas que han demostrado su buena adaptación a las condiciones impuestas, pudiendo utilizarse para fines de alimentación animal (Capim Elefante de las variedades Mercker y Napier, Capim Sempre-Verde, Cana-de-açúcar) y Capim Vetiver, el cual también posee otras utilidades como la fabricación de perfumes, artesanía y utensilios domésticos (escobas, alfombras y esteras).

En términos de recomendación para futuras investigaciones, se sugiere utilizar otras especies vegetales para pruebas de adaptabilidad al ambiente salinizado de la presa subterránea. Esas especies pueden ser fructíferas, ornamentales y gramíneas de potencial forrajero. Otra sugerencia sería la realización de la comparación de la producción de las especies probadas en ambiente salinizado de la presa con otros ambientes sin ese problema. Para evitar problemas de salinización del agua y suelo del depósito en presas subterráneas es fundamental colocar un tubo de descarga de fondo, sobre la capa impermeable, saliendo de aguas arriba, perforando el macizo hasta aguas abajo, donde en esta extremidad se instala una curva de $90^{\circ}$ con otro tubo en la vertical que funcionará como un pozo, pudiendo bombearse o desaguarse esta agua sobre el suelo. Este tubo permitirá, periódicamente, el lavado del perfil del suelo llevando las sales disueltas en el agua del depósito subterráneo.

\section{REFERENCIAS}

Almeida, S. Pinhão-manso, a opção para o biodiesel. Comunicado técnico. Disponible en http://www.pinhaomanso.com.br/noticias/jatropha/pinhao_manso_opcao_biodiesel_18_04_07.html . Acesso: 20 agosto (2009).

Anjos, J. B.; Silva, M. S. L. DA. Equipamento para o monitoramento do nível de lençol freático em áreas com barragens subterrâneas. Petrolina-PE: Embrapa Semi-Árido. Comunicado técnico, $\mathrm{n}$ 132 (2007).

Apha., Standard methods for examination of water and wastewater, 18th edition. Washington, DC. American Public Health Association (1992).

Ayers, R. S. y Westcott, D. W. A qualidade da água na agricultura. Estudos FAO: Irrigação e drenagem, 29, Campina Grande: UFPB (1991).

Bastos, R. K. X. Utilização agrícola de esgotos sanitários, Apostila, São Paulo: Associação Brasileira de Engenharia Sanitária e Ambiental (ABES) (1999).

Bernardo, S. Manual de irrigação. 6ª edição, Viçosa, MG, UFV, Imprensa Universitária (2006).

Bouwer, H. y Idelovitch, E. Quality requirements for irrigation with sewage water. Journal of irrigation and drainage engineering, v. 113, (4), 516-533 (1988).

Cabral, J.J.S.P; Lobo Ferreira, J.P.C; Montenegro, S.M.G.L; Costa, W.D., 2004. Água Subterrânea Aqüíferos Costeiros e Aluviões, Vulnerabilidades e Aproveitamento. Recife: Editora Universitária da UFPE (2004).

Chaves, V. C.; Ferreira, G. B.; Mendonça, C. E. S.; Alberto, C.; Silva, M. S. L. da. Caracterização de solos de quatro barragens subterrâneas: I. Atributos físicos e químicos. En: CONGRESSO BRASILEIRO DE AGROECOLOGIA, 5, 2007, Espírito Santo - ES. Anais Revista Brasileira de Agroecologia (2007).

Cordeiro, G. Salinidade em áreas irrigadas. Petrolina: Empresa Brasileira de Pesquisa Agropecuária (EMBRAPA SEMI-ÄRIDO) (2002). 
Cirilo, J.A. y Costa, W.D., Barragem subterrânea: uma alternativa a considerar, Revista Informativa da Associação Brasileira de Recursos Hídricos, (2), jul/2006, (2006).

Costa, W.D., "Riscos Potenciais E Reais Decorrentes Da Super-Exploração Das Águas Subterrâneas No Recife PE" Anais en CD del $1^{\circ}$ Congreso Mundial Integrado de Aguas Subterráneas ABAS/ALSHUD/IAH Fortaleza 31 julho el 4 agosto (2000).

Ferreira, G. B.; Silva, M. S. L. da; Mendonça, C. E. S.; Ribeiro, F. N.; Anjos, J. B. dos \& Cunha, T. J. F. Barragem subterrânea uma inovação de transformação social para a agricultura familiar. En: Reunión Brasileña De Fertilidad Del Suelo Y Nutrición De Plantas, 28, 2008, Londrina, 2008. Anais. Londrina-PR, SBCS, CD-ROM 43 (2008).

Gomes, C.C., "Modelagem matemática para estudo de comportamento de aqüífero aluvial represado por barragem subterrânea", Disertación de maestría, Universidade Federal do Ceará, Fortaleza - CE (1990).

Embrapa. Manual de métodos de análise de solos. $2^{\mathrm{a}}$ edición, Rio de Janeiro: Embrapa-MA (1997).

Mendonca, C. E. S; Chaves, V. C.; Ferreira, G. B.; Mendes, A. M. S.; Silva, M. S. L. da. Monitoramento de atributos químicos e físicos de solos de barragens subterrâneas em dois estados do Nordeste. En: Congreso Brasileño De Agroecología, 5, Espírito Santo - ES. Anais Revista Brasileira de Agroecologia (2007).

Metcalf \& Eddy. Wastewater engineering treatment, disposal and reuse. 3 ed. New York: McGraw Hill, (1991).

Miranda, J.R.P. Silício e cloreto de sódio na nutrição mineral e produção de matéria seca de plantas de Cajueiro Anão-Precoce (Anacardium occidentale L.) e de Moringa (Moringa oleifera Lam.). Lavras: Universidade Federal de Lavras, tese de doutoramento, 186 p. (2000).

Monteiro, Luiz Cristiano Campos, "Armazenamento de água em barragens subterrâneas", Disertación de maestría, Universidade Federal do Ceará, Fortaleza - CE (1988).

Pereira, J. R.; Valdivieso, C. R.; Cordeiro, G. G. Recuperação de solos afetados por sais através do uso do gesso. En: Seminario sobre el uso del fosfo-yeso en la agricultura, Brasília, p.85-105 (1985).

Rebouças, A.C., Águas subterrâneas. En: Aguas dulces en Brasil; capital ecológico, uso y conservación, organización y coordinación científica, Rebouças, A.C., Braga, B. e Tundisi, J.G., Escrituras Editora (1999).

Reichart, K., A água na produção agrícola. Campinas: McGraw Hill (1978).

Saturnino, H.M. Pacheco; Kakido, J. Tominaga, N.; Gonçalves, N.P., Cultura do pinhão-manso (Jatropha curcas L.) En: Informe agropecuario: Producción de oleaginosas para biodiesel, Belo Horizonte, v.26, (229), 44-78 (2007).

Silva, M. S. L. Da; Oliveira Neto, M. B. De; Ferreira, G. B.;. Aspectos técnicos e sociais sobre barragem subterrânea. En: Reunión Brasileña De Manejo Y Conservación Del Suelo Y Del Agua, 17, 2008, Rio de Janeiro. Anais. Rio de Janeiro, SBCS (2008).

Silveira, J. W., "Aproveitamento de efluentes de estações de tratamento de esgotos de indústrias têxteis". Tesis de doctorado. Universidade Federal do Ceará, Fortaleza - CE (2003). 